\title{
Particle Nature of Photons, Straight Diffraction Pattern and Curved Diffraction Pattern Emerging in Same Grating Experiment
}

Hui Peng ( $\nabla$ davidpeng1749@yahoo.com )

\section{Research Article}

Keywords: particle nature of photons, wave-particle duality, Bohr's complementarity principle, diffraction pattern, straight diffraction pattern, curved diffraction pattern, grating experiment, Feynman's mystery, grating

Posted Date: October 4th, 2021

DOl: https://doi.org/10.21203/rs.3.rs-952063/v1

License: (c) (1) This work is licensed under a Creative Commons Attribution 4.0 International License. Read Full License 


\title{
Particle Nature of Photons, Straight Diffraction Pattern and Curved Diffraction Pattern Emerging in Same Grating Experiment
}

\author{
Hui Peng \\ Davidpeng1749@gmail.com
}

\begin{abstract}
The particle nature of the photons was experimentally confirmed. The static straight line diffraction pattern of the normal grating experiments has been shown experimentally. The phenomenon of the dynamic curved diffraction pattern of the grating experiment have been shown in separate experiments. In this article, the new experiments are proposed and performed, which show that the particle nature of the photons, the static straight line diffraction patterns, and the dynamic curved, expanded and inclined diffraction patterns co-exist in the same grating experiment simultaneously. The novel phenomena make the Feynman's mystery of the normal double slit experiment more mysterious, violate Bohr's complementarity principle, and provide comprehensive information/data for studying the wave-particle duality and developing new theoretical model.
\end{abstract}

Keywords: particle nature of photons, wave-particle duality, Bohr's complementarity principle, diffraction pattern, straight diffraction pattern, curved diffraction pattern, grating experiment, Feynman's mystery, grating

Declaration: There is not interesting conflict.

\section{Introduction}

Young's 1D-double slit experiment was performed in 1801 [1] [2]. The experiments create the interference pattern. The fringes of the interference pattern distribute along straight line. For the certain double slit, the interference pattern is static, i.e., the style of the interference pattern and the spacing between the fringes do not change. Feynman stated that "In reality, it [double slit experiment] contains the only mystery [of quantum mechanics]" [3].

Both the 1D-double-slit experiment and the photoelectric effect led to wave-particle duality and Bohr's complementarity principle. It is concluded that photons can behave as either particles or waves, but cannot be detected as both at the same experiment. The which way double slit experiments were considered as the confirmation of the complementarity principle. Namely, the particle nature and wave nature cannot manifest in the same experiment at the same time.

Recently, the delayed-choice experiments [4] [5] and which-way experiment [6] [7] show that the complementarity principle is violated.

Recently, the experiments show the phenomena that the dynamic interference/diffraction patterns are curved, expanded and inclined continuously [8] [9] [10].

In this article, to study the wave-particle duality, Bohr's complementarity principle and Feynman's mystery of the double slit experiment, we propose and perform new experiments that show that:

(1) the particle nature and wave distribution are manifested in the same experiments;

(2) the complementarity principle is violated; 
(3) the static straight diffraction patters and the dynamic curved diffraction patterns co-exist in the same experiment simultaneously.

The nature of photons really puzzled Einstein. He wrote to M. Besso: "All these 50 years of conscious brooding have brought me no nearer to the answer to the question: What are light quanta?" [11].

The new experiments provide comprehensive information/data for understanding the waveparticle duality and for developing new theoretical model.

\section{Experimental Setups}

First let us define several terms.

1) "Same experiment": In an experiment, if only one source of light beam and only one grating is utilized, we referred it as the "same experiment". Since the beam-splitter (BS) and mirror do not change the nature of the light beam, the experiment may utilize BS and mirror.

2) "Original Orientation of the grating": the orientation, at which the light beam is perpendicular to the plane of the grating, i.e., the normal vector of the plane of the grating points to the source of the light, is referred as the "original orientation" of the grating.

The grating contains multi-slits. In Figure 1, we only draw two slits to represent the grating, and the grating is at its original orientation.

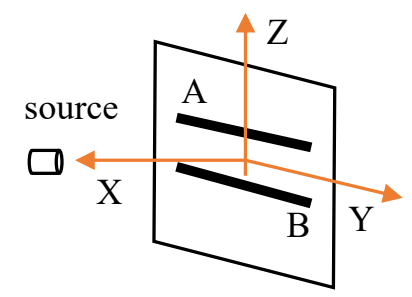

Figure 1 Grating in Right-hand Coordinate

The following were shown [9] [10]: (1) when the grating rotates around the Z-axis, the diffraction pattern is curved; (2) when the grating rotates around Y-axis, the diffraction pattern is expanded; (3) when the diaphragm of the double slit/cross-double slit rotates around $\mathrm{X}$-axis, the interference pattern has the rotation-symmetry, namely the characteristics of the interference pattern does not change, only the direction of the interference pattern is changed (Figure 1). It is reasonable to state that the diffraction pattern of the grating have the same rotation symmetry (which will be shown in the following experiment-1 and -2).

First, let us rotate the grating around Z-axis, clockwise and counterclockwise respectively, at a predetermine angle, for example, 75 degrees, then continuously rotate the grating around $\mathrm{X}$-axis.

Two experimental setups are shown in Figure 2 and Figure 3.

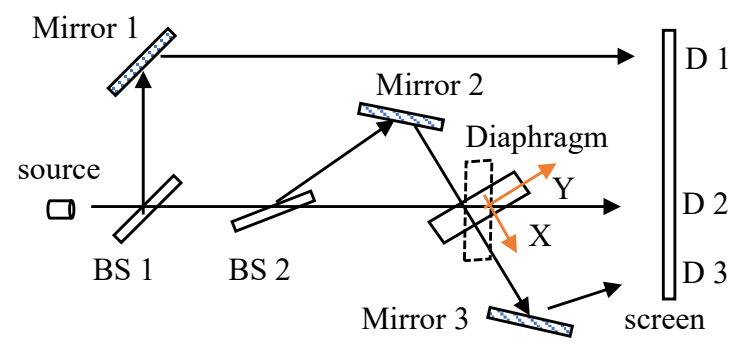

Figure 2 Grating rotates around $\mathrm{Z}$ axis clockwise

First experimental setup (Figure 2): the grating is rotated clockwise around Z-axis at a predetermined 
angle. We divide the laser beam into three sub-beams:

1) Beam-1: travels from the source to BS1 to mirror1 and lands at D1 on the screen;

2) Beam-2: travels from the source to BS1 to BS2, passes the grating and lands at $D 2$ on the screen;

3) Beam-3: travels from the source to BS1 to BS2 to mirror 2, passes the grating, reflected by mirror 3 and lands at D3 on the screen.

Second experimental setup (Figure 3): the grating is rotated counterclockwise around Z-axis at a predetermined angle. We divide the laser beam into three sub-beams:

(1) Beam-1: travels from the source to BS1 to mirror1 and lands at D1 on the screen;

(2) Beam-2: travels from the source to BS1 to BS2, passes the grating and lands at D2 on the screen;

(3) Beam-3: travels from the source to BS1 to BS2 to mirror 2, passes the grating, reflected by mirror 3 and lands at $\mathrm{D} 3$ on the screen.

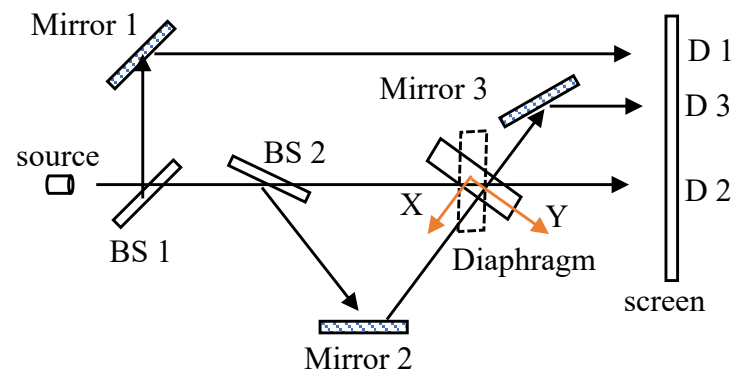

Figure 3 Grating rotate around $\mathrm{Z}$ axis counterclockwise

Note that, in both setups, when travel from mirror 2 to mirror 3 , beam-3 is perpendicular to the plane of the grating.

\section{Experiments}

Experiment-1 (Figure 2): Rotating the grating around $\mathrm{Z}$ axis 75 degree clockwise and stay.

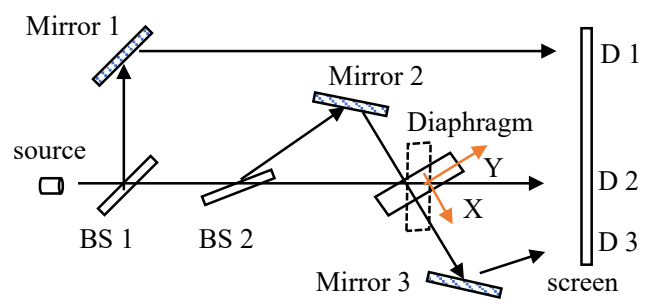

Figure 2 First experimental setup

We perform the experiment in two steps.

First step (Figure 4): Rotate the grating around $\mathrm{X}$ axis clockwise

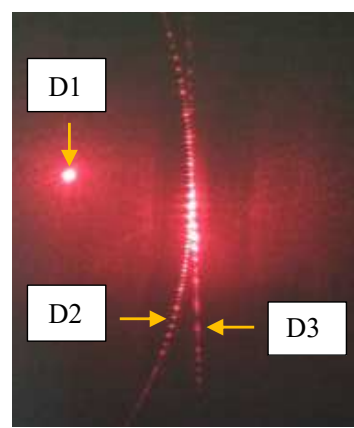

. (a) Original pattern

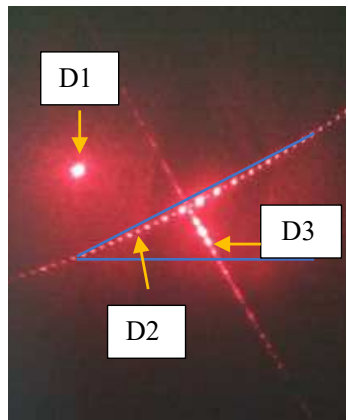

(b) rotate 65 degrees

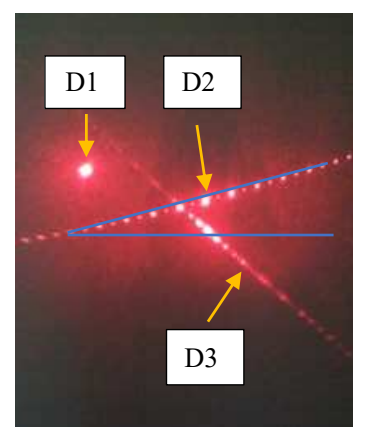

(c) rotate 75 degrees

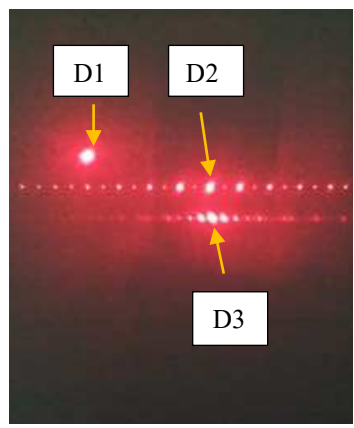

(d) rotate 90 degrees

Figure 4 Patterns due to rotating grating clockwise around $\mathrm{X}$ axis

\section{Observation:}

Figure 4a shows the original patterns. Beam-1 is the image of the source, which shows the particle 
nature of photons. Attributing to the rotation of the grating around $\mathrm{Z}$ axis clockwise, which obey the Right-hand rule, beam-2 forms curved pattern towards to left. Since beam-3 always travels to the grating at the right angle, so it always forms the normal straight pattern.

Figures 4b, 4c and 4d show: When the grating rotates around $\mathrm{X}$-axis clockwise at different angles,

(1) the curved pattern starts to incline toward to the horizontal line;

(2) the spacings between fringes start to expand;

(3) the curvature of the curved patter becomes smaller and smaller;

(4) the curved pattern rotates clockwise;

(5) the straight-line pattern rotates counterclockwise, which is due to Mirror 3.

When the grating rotates 90 degrees, the curved pattern becomes straight line pattern, the expansion reaches the largest, the pattern is in the horizontal direction.

Conclusion: The particle nature of the photons, the static straight-line pattern and the dynamic curved pattern coexist in the same experiment.

Second Step (Figure 5): Rotate the grating around X axis counterclockwise:

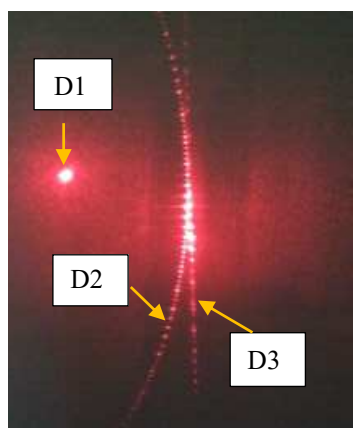

.(a) Original pattern

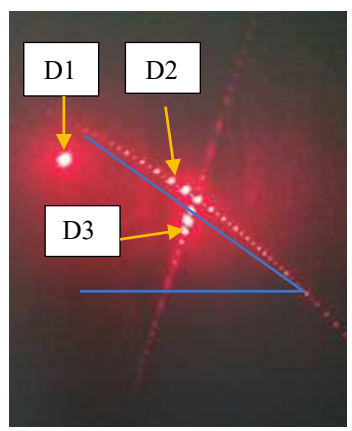

(b) Rotate 55 degrees

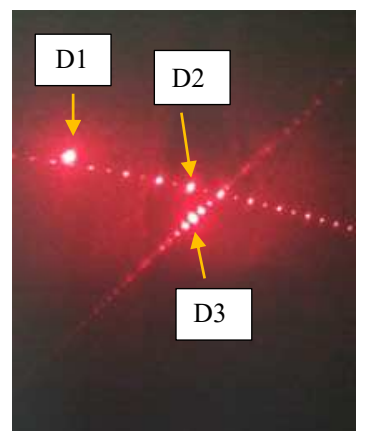

(c) Rotate 75 degrees

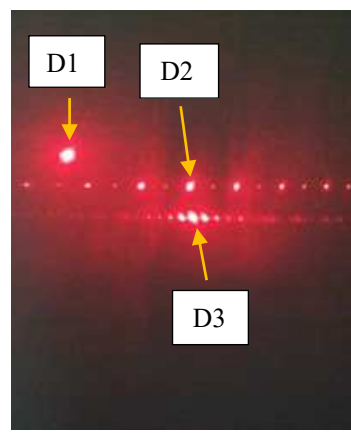

(d) Rotate 90 degrees

Figure 5 Patterns due to rotating grating counterclockwise around $\mathrm{X}$ axis

\section{Observation:}

Figure 5a shows the original patterns. Beam-1 is the image of the source, which shows the particle nature of photons. Beam-2 forms curved pattern towards to left. Beam-3 forms the normal straight pattern.

Figures 5b, 5c and 5d show: When the grating rotates around $\mathrm{X}$-axis counterclockwise at different angles,

(1) the curved pattern starts to incline toward to the horizontal line;

(2) the spacings between fringes start to expand;

(3) the curvature of the curved patter becomes smaller and smaller;

(4) the curved pattern rotates counterclockwise;

(5) the straight-line pattern rotates clockwise, which is due to Mirror 3.

When the grating rotates 90 degrees, the curved pattern becomes straight line pattern, the expansion reaches the largest, the pattern is in the horizontal direction.

Conclusion: The particle nature of the photons, the static straight-line pattern and the dynamic curved pattern coexist in the same experiment.

Experiment-2 (Figure 3): Rotating the grating around $\mathrm{Z}$ axis 75 degree counterclockwise and stay.

Then we perform the experiment in two steps. 


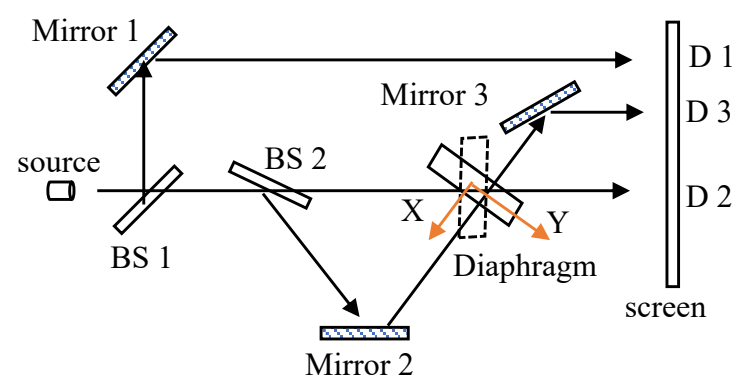

Figure 3 Second experimental setup

First step (Figure 6): Rotate the grating around $\mathrm{X}$ axis counterclockwise

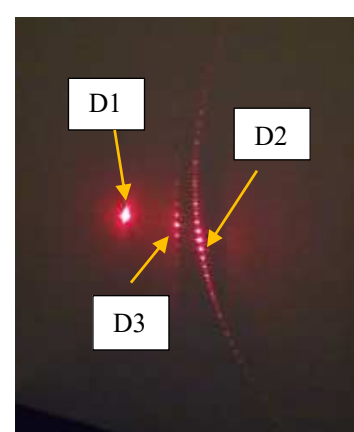

.(a) Original patterns

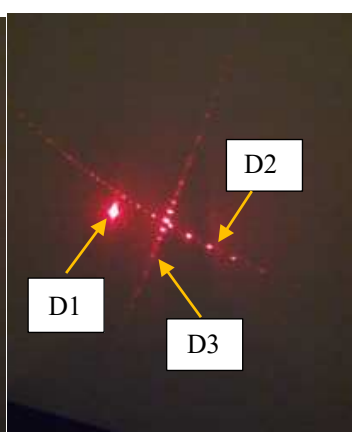

(b) rotating 45 degrees

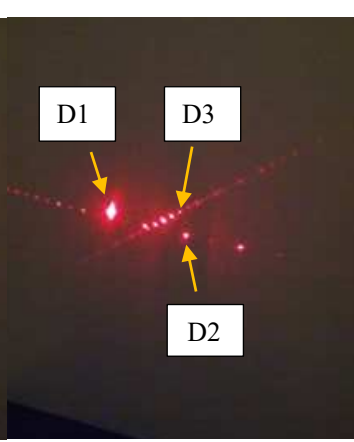

(c) rotating 75 degrees

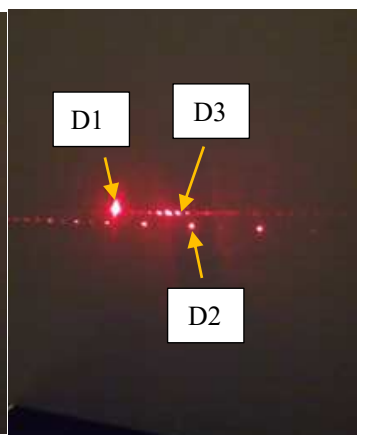

(d) rotating 90 degrees

Figure 6 Patterns due to rotating grating counterclockwise around $\mathrm{X}$ axis

Observation: Figure 6a shows the original patterns. Beam-1 is the image of the source, which shows the particle nature of photons. Beam-2 forms curved pattern towards to right. Beam-3 forms the normal straight pattern.

Figures $6 \mathrm{~b}, 6 \mathrm{c}$ and $6 \mathrm{~d}$ show: When the grating rotates around $\mathrm{X}$-axis counterclockwise at different angles,

(1) the curved pattern starts to incline toward to the horizontal line;

(2) the spacings between fringes start to expand;

(3) the curvature of the curved patter becomes smaller and smaller;

(4) the curved pattern rotates counterclockwise;

(5) the straight-line pattern rotates clockwise, which is due to Mirror 3.

When the grating rotates 90 degrees, the curved pattern becomes straight line pattern, the expansion reaches the largest, the pattern is in the horizontal direction.

Conclusion: The particle nature of the photons, the static straight-line pattern and the dynamic curved pattern coexist in the same experiment.

Second step (Figure 7): Rotate the grating around X axis clockwise

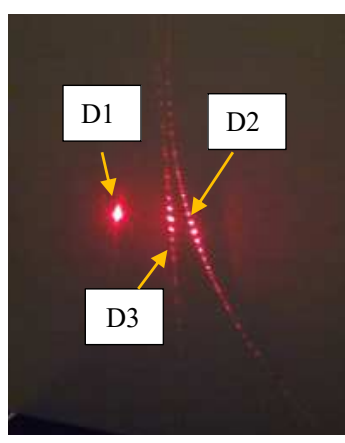

(a) Original patterns

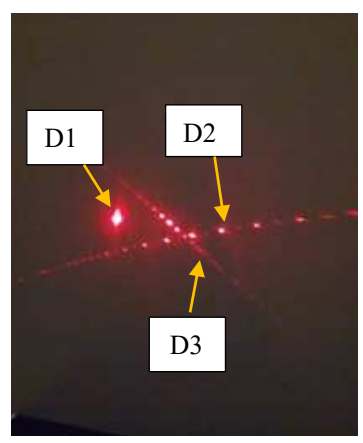

(b) rotating 75 degrees

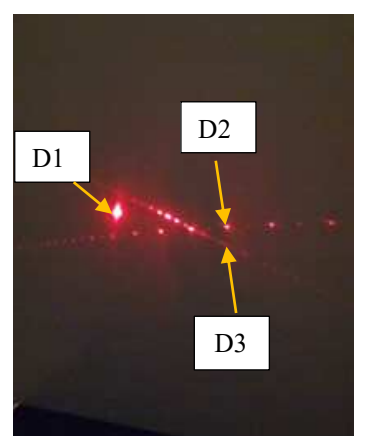

(c) rotating 80 degrees

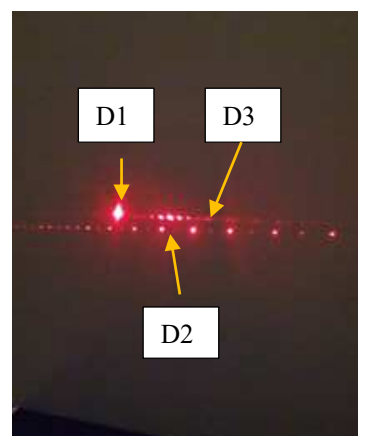

(d)rotating 90 degrees

Figure 7 Patterns due to rotating grating clockwise around $\mathrm{X}$ axis 


\section{Observation:}

Figure 7a shows the original patterns. Beam-1 is the image of the source, which shows the particle nature of photons. Beam-2 forms curved pattern towards to right. Beam-3 forms the normal straight pattern.

Figures $7 \mathrm{~b}, 7 \mathrm{c}$ and $7 \mathrm{~d}$ show: When the grating rotates around $\mathrm{X}$-axis clockwise at different angles,

(1) the curved pattern starts to incline toward to the horizontal line;

(2) the spacings between fringes start to expand;

(3) the curvature of the curved patter becomes smaller and smaller;

(4) the curved pattern rotates clockwise;

(5) the straight-line pattern rotates counterclockwise, which is due to Mirror 3.

When the grating rotates 90 degrees, the curved pattern becomes straight line pattern, the expansion reaches the largest, the pattern is in the horizontal direction.

Conclusion: The particle nature of the photons, the static straight-line pattern and the dynamic curved pattern coexist in the same experiment.

\section{Discussion and Conclusion}

The particle nature of photons exists throughout the experiments. The curved, inclined and expanded patterns exist. The curvature, the inclination angle and the spacings between the fringes are continuously change while the grating continuously rotates. The normal straight pattern exists throughout the experiments.

It is difficult to explain those experiment consistently.

Those experiments lead to restudy the complementarity principle and the wave-particle duality.

The novel phenomena make the Feynman's mystery of the normal double slit experiment more mysterious.

Note that the experiment- 1 and experiment- 2 may be done with the diaphragms of the double slit and the cross-double slit. The phenomena are the same. The grating experiments are the easiest to perform. Also, it is interesting to perform the experiments by emitting photons one at the time.

\section{References}

[1] A. Ananthaswamy, "Through Two Doors at Once", Dutton, New York, NY, 2018.

[2] A. Robinson, "The Last Man Who Knew Everything". New York, NY: Pi Press., 2006.

[3] R. Feynman, R. Leighton, and M. Sands, “The Feynman Lectures on Physics” (Addison-Wesley, Reading), Vol. 3, 1966.

[4] Hui Peng, "Present Does Not Decide Past Retroactively---Novel Delayed-Choice Experiments". Inter. J. of Phys., vol. 9, no. 1: 48-52. DOI: 10.12691/ijp-9-1-6, 2021.

[5] Hui Peng, "Extended-Delayed-Choice Experiments Supporting Causality". hal-03354391, 2021

[6] Hui Peng, "Observation of Which-Way-2D-Cross-Double-Slit Experiments: Violation of Bohr's Complementarity Principle.” Inter. J. of Phys., vol. 8, no. 4: 153-157. DOI: 10.12691/ijp-8-4-6, 2020.

[7] Hui Peng. Extended-Which-Way Experiments: Violating Complementarity Principle. hal- 03357688, 2021.

[8] Hui Peng, "Curved Interference Patterns---Novel Double Slit and Cross-double Slit Experiments", Research Square, preprint, DOI: https://doi.org/10.21203/rs.3.rs-677223/v1, 2021.

[9] Hui Peng, "Straight Interference Pattern to Curved-Expanded- Inclined Interference Pattern--- 
Static to Dynamic Double Slit/Cross-Double Slit Experiments", Research Square, preprint, DOI: https://doi.org/10.21203/rs.3.rs-846260/v1, 2021.

[10] Hui Peng, "Curved, Expanded and Inclined Interference Patterns---

Dynamic Double Slit/Cross-Double Slit Experiments", hal-03338628, 2021.

[11] S. Rashkovskiy, "Is a rational explanation of wave-particle duality possible?" arXiv 1302.6159 [quant-ph], 2013. 